\title{
INCREASING INCIDENCE OF TUBERCULOSIS DIAGNOSED BY SURGERY: A SINGLE CENTRE ANALYSIS IN LOW-INCIDENCE COUNTRY
}

\author{
Radek Pohnán ${ }^{1,2}$, Vladislav Hytych ${ }^{2}$, Ivana Holmquist ${ }^{3,4}$, Vanda Boštíková4, Radek Doležel ${ }^{1}$, Miroslav Ryska ${ }^{1}$ \\ 'Department of Surgery, Second Faculty of Medicine, Charles University and Central Military Hospital, Prague, Czech Republic \\ ${ }^{2}$ Thomayer Hospital, Prague, Czech Republic \\ ${ }^{3}$ Emory University Hospital Midtown, Atlanta, Georgia, USA \\ ${ }^{4}$ Department of Epidemiology, Faculty of Health Sciences, University of Defence, Hradec Králové, Czech Republic
}

\begin{abstract}
SUMMARY
Objective: The aim of this study was to assess the incidence of thoracic tuberculosis (TB) in patients who underwent surgery for indeterminate lung nodules, mass and pleural effusions.

Methods: A monocentric retrospective study was carried out from 2012 to 2018 in a high-volume thoracic surgery centre. All patients with finding of thoracic TB within surgery and/or confirmed post-surgery were studied. Demography, origin, TB related symptoms, immunosuppression, type of surgery, and complication of surgery were analyzed.

Results: During the seven-year period TB was diagnosed in 71 cases, $58 \%$ were men. The mean age was 50 years. $21 \%$ of the cases had family history of TB or were successfully treated for TB in the past. $14 \%$ of patients had prior history of treatment for malignancy. Five patients $(7 \%)$ received immunosuppressive therapy. The indication for surgery was indeterminate lung nodules and mass in 55 patients $(77.5 \%)$ and indeterminate recurrent or persistent pleural effusions in 21 patients (22.5\%). In five patients (7\%) a lung carcinoma and a concomitant TB infection was detected. 63 of the cases (88.7\%) had positive real-time PCR TBC test. Direct microscopic detection of Mycobacterium tuberculosis detected TB in five cases (7\%). The microbiological diagnosis by culture was achieved in 19 patients $(26.8 \%)$. Two patients $(2.8 \%)$ were diagnosed with multidrug-resistant TB. Surgical procedure complications occurred in nine cases (12.7\%).

Conclusions: Although the overall incidence of TB in the Czech Republic is low and constantly continues to decrease, the number of TB detected by surgical procedures is increasing. Surgery still remains an important tool in diagnostics of nonobvious cases of TB, especially in patients with a potential risk of malignancy.
\end{abstract}

Key words: tuberculosis, surgical procedures, epidemiology, incidence, diagnostics

Address for correspondence: V. Boštiková, Faculty of Military Health Sciences, University of Defence in Hradec Králové, Třebešská 1575, 50001 Hradec Králové, Czech Republic. E-mail: vanda.bostikova@unob.cz

https://doi.org/10.21101/cejph.a5789

\section{INTRODUCTION}

Tuberculosis (TB) is a global disease that is the most common cause of death caused by an infectious disease worldwide (1). TB continues to pose a global threat despite the fact that the causing agent was discovered 137 years ago, and an effective antibiotic therapy is available for more than half a century.

The Czech Republic has low incidence of this disease that continues to decrease. In 2017, a total of 505 TB cases of all forms and localizations (4.8 cases/100,000 population) were reported to the TB Register of the Czech Republic (in 2016 it was 517 cases) (2).

Decreasing incidence of TB in the Czech Republic is a result of effective control of this infectious disease. Geographic location, high standard of living, functional treatment system and strict legal regulations govern the treatment of suspected and confirmed
TB cases. A significant contribution to the TB incidence in the Czech Republic is the historically extensive experience with this infectious disease (3-6).

Thoracic tuberculosis can present a challenge for clinicians, although in most cases the diagnosis is based on a combination of imaging methods, microbiological and histopathological examination, and epidemiological context $(3,5)$.

Surgery has been a unique tool of TB management for several decades. However, with introduction of antituberculous therapy (ATT) the role of surgery became limited to the treatment of TB complications and diagnoses of uncertain lesions or otherwise unrecognized disease (7-11).

The current global rise of TB, the emergence of multi-resistant forms of TB (MDR-TB) and increase of immunosuppressed patients pose new challenges for the surgical diagnostics and treatment of TB $(9,12)$. 
The purpose of this work was to analyze the incidence of TB captured during surgery for indeterminate lung nodules, mass and pleural effusions.

\section{MATERIAL AND METHODS}

This study was a retrospective analysis of a prospectively maintained database of all patients admitted to the Department of Thoracic Surgery (DTS) at the Thomayer Hospital in Prague in the Czech Republic. The DTS is part of the Centre for Treatment of Pulmonary and Extra-pulmonary Tuberculosis and Mycobacteriosis, regional TB treatment centre. Patients enrolled in this study underwent comprehensive preoperative examination and lab work and then surgery for indeterminate lung nodules, mass or pleural effusions, and were diagnosed with TB intraoperatively or post-surgically.

Demographics, socioeconomic characteristic, anamnesis, TB related symptoms, immunosuppression, indications for surgery, surgical procedure, complication of surgery, histopathologic and microbiologic findings, and patients' outcomes were analyzed.

TB diagnosis was based on the detection of Mycobacterium tuberculosis complex in a culture assay. Microscope was used for direct detection of mycobacteria and histopathologic evaluation was completed to identify TB specific granuloma. Both detection methods were confirmed by the TB PCR positivity (real-time PCR TBC test).

Data were collected from a seven-year period between 1 January 2012 and 31 December 2018 (Fig. 1). Data were stored in an Excel spreadsheet (Microsoft Corp., Redmond,WA, USA) and analyzed with the Stata13 software (StataCorp, USA). The two-sample t-test was performed for operative time. The Mann-Whitney U test was used for postoperative stay and blood loss. The Fisher's exact test was used for post-operative complications. Statistical significance was set at $\mathrm{p}<0.05$.

\section{RESULTS}

\section{Clinical Presentation}

Between January 2012 and December 2018, 960 patients underwent surgery for indeterminate lung nodules, mass and pleural effusions. From this cohort a group of 71 cases, where TB was consecutively identified, was selected. There were 41 males $(58 \%)$ and 30 females $(42 \%)$. The median age was 50 years (range 19-79 years).

In nine cases $(12.7 \%)$ there was positive TB family history, six patients $(8.4 \%)$ were successfully treated with ATT for TB in the past (range 10-35 years prior to surgery). Ten patients (14\%) had prior history of complex oncological treatment for malignancy (range 1-12 years prior to thoracic surgery). Two patients (2.8\%) received immunosuppressants due to kidney transplantation. Three patients $(4.2 \%)$ with rheumatoid arthritis were treated by corticoid medication. Pharmacological immunosuppression and a history of malignancies were associated with severe stage in operations for pleural effusion $(p=0.004)$. The most frequent symptom was cough, which was described by 41 of patients $(58 \%)$. Five patients $(7 \%)$ suffered from thoracic pain, another

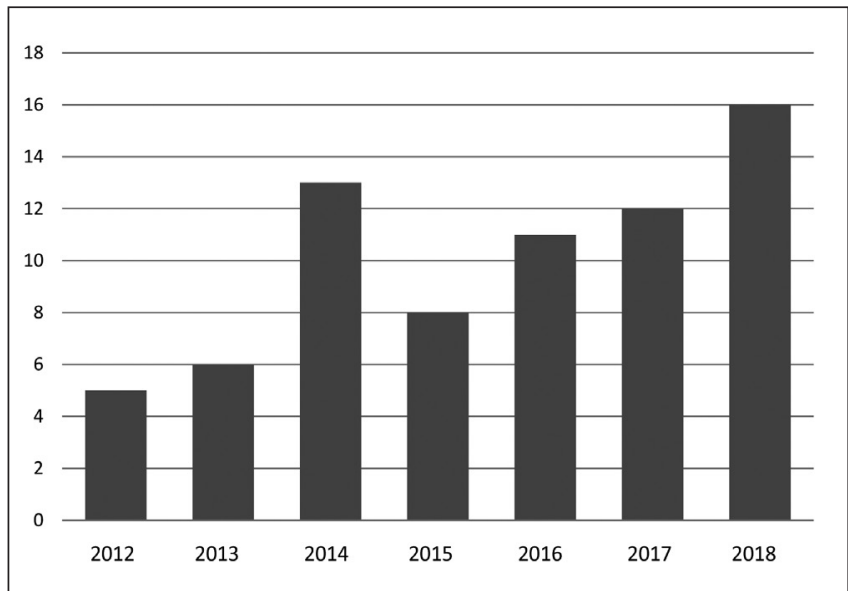

Fig. 1. Incidence of TB diagnosed by surgery between 20122018, Department of Thoracic Surgery, Thomayer Hospital in Prague, Czech Republic.

five patients had a weight loss. Also loss of appetite was recorded in five of the cases $(7 \%)$. Total 22 patients $(30.9 \%)$ had no symptoms. Clinical and demographic profiles are displayed in Table 1.

\section{Socioeconomic Characteristics}

Twelve foreign nationals (persons born outside the Czech Republic) accounted for $16.9 \%$ of the total number of identified TB cases. Four of these people were citizens of Ukraine (5.6\%). Three patients were from Slovakia (4.2\%), another three patients

Table 1. Clinical profile and origin of patients with TB $(N=71)$

\begin{tabular}{|l|c|}
\hline Age (years) & $19-79$ \\
\hline Range & $50.5 \pm 16.3$ \\
\hline Mean \pm SD & $41 / 30(58 / 42 \%)$ \\
\hline Gender \\
\hline Male/female \\
\hline Demographics \\
\hline Citizens & $59(83.1 \%)$ \\
\hline Foreigners & $12(16.9 \%)$ \\
\hline Country of origin & $59(83.1 \%)$ \\
\hline Czech citizens & $4(5.6 \%)$ \\
\hline Ukraine & $3(4.2 \%)$ \\
\hline Slovakia & $3(4.2 \%)$ \\
\hline Vietnam & $3(4.2 \%)$ \\
\hline Romania & \\
\hline History pertaining to tuberculosis & $9(12.7 \%)$ \\
\hline Positive family history & $6(8.4 \%)$ \\
\hline Prior history & $10(14 \%)$ \\
\hline History pertaining to malignancy & $3(4.2 \%)$ \\
\hline History pertaining to immunosuppression & \\
\hline Prior history & \\
\hline Organ transplanted & $(7 \%)$ \\
\hline Corticotherapy & \\
\hline
\end{tabular}


came from Vietnam (4.2\%), and two from Romania (2.8\%). All these patients were labourers with elementary education. 59 patients (83.1\%) were Czech citizens. Two of them (2.8\%) finished elementary education. A total of $50(70.4 \%)$ patients completed secondary school. Seven patients $(9.9 \%)$ graduated from university. 35 patients were $(49.3 \%)$ employed, nine patients $(12.7 \%)$ were retired, seven patients $(9.9 \%)$ were supported persons and eight patients $(11.3 \%)$ were unemployed, one patient was a homeless person $(1.4 \%)$.

\section{Surgical Procedure and Outcomes}

The indication for surgery was indeterminate lung nodules and mass in 55 patients $(77.5 \%)$ and indeterminate recurrent or persistent pleural effusions in 16 patients $(22.5 \%)$. Details of surgery are depicted in Table 2 . Out of the 71 patients, 43 $(60.6 \%)$ underwent a minimally invasive surgical procedure and $28(39.4 \%)$ had thoracotomy surgery. There was no significant difference in the duration of surgery $(p=0.78)$ and blood loss $(p=0.178)$ in the minimally invasive surgery group compared to the open surgery group.

In five cases the pathologist found lung carcinoma in the preoperative rapid frozen section biopsy. Lung lobectomy, with systematic mediastinal lymfadenectomy, was performed. Concomitant TB infection was detected in all five cases.

A total of 50 (70.4\%) patients were treated with non-anatomic wedge lung resection. Minimally invasive video-assisted thoracoscopic surgery (VATS) approach was used in 33 of the cases $(66 \%)$. Total 37 patients $(74 \%)$ had a solitary lesion, eight patients (16\%) had two lesions and 5 patients $(10 \%)$ had three lesions.

Sixteen patients $(22.5 \%)$ underwent surgery for indeterminate pleural effusion. In seven patients there was evacuated pleural effusion and a pleural biopsy was performed using the thoracoscopic approach. In nine cases (12.7\%) severe empyema was found, therefore, thoracotomy, pleurectomy and lung decortication was performed. The mean post-operative stay at the surgery department was 7.1 \pm 5.1 (standard deviation) days. The significant improvement in shorter post-operative stay at the surgery department was observed in the minimally invasive surgery group $(p=0.002)$.
The incidence of post-operative complications was $12.7 \%$ $(\mathrm{n}=9)$. The most common complication was persistent air leak $(n=2)$, pneumonia $(n=2)$, and atelectasis $(n=2)$. Other complications included surgical site infection $(n=1)$, post-operative hemorrhage $(n=1)$, and respiratory failure $(n=1)$. Patients treated for indeterminate pleural effusion were more likely to have postoperative complications $(\mathrm{p}=0.04)$.

\section{Histopathologic Examination and Confirmation of TB}

Specimens from all 71 patients were subjected to histopathologic examination. Caseating granuloma was found in 42 patients $(59.2 \%)$ and non-necrotizing granulomas in 24 cases (33.8\%). Synchronous lung malignancy was detected in five patients. Lung adenocarcinoma was diagnosed in three cases $(4.2 \%)$ and epidermoid carcinoma was revealed in two cases $(2.8 \%)$.

The real-time PCR TBC test confirmed TB diagnoses in 63 cases $(88.7 \%)$. Direct microscopic detection of Mycobacterium tuberculosis detected TB in 5 patients (7\%). Microbiological diagnosis by culture confirmed TB in 19 patients $(26.8 \%)$. Two patients were diagnosed with MDR-TB.

\section{Follow-up}

After detection of TB and the removal of the thoracic drain, all patients were transferred to the department of pulmonary diseases and tuberculosis for long-term antibiotic treatment. There were no late complications or death due to surgical treatment. Four patients are still receiving ATT. Ten patients (14\% - all foreigners) did not return for follow up care. 61 patients $85.9 \%$ complied with the follow up care. The median length of follow up care was 27 months (4-48 months).

\section{DISCUSSION}

The diagnosis of TB is usually based on a combination of anamnesis, imaging methods and detection of Mycobacterium tuberculosis in the sputum, pleural fluid or biopsy specimens (3, $5,12)$. Nevertheless, the TB diagnosis can present a challenge for

Table 2. Details of surgery and surgical outcomes $(N=71)$

\begin{tabular}{|c|c|c|c|}
\hline & $\begin{array}{l}\text { Minimally invasive surgery group } \\
\qquad n=43(60.6 \%)\end{array}$ & $\begin{array}{l}\text { Open surgery group } \\
\qquad n=28(39.4 \%)\end{array}$ & $p$-value \\
\hline \multicolumn{4}{|l|}{ Surgery details (n) } \\
\hline Non-anatomical lung resection & 33 & 17 & \multirow{3}{*}{-} \\
\hline Lobectomy & 3 & 2 & \\
\hline Pleural effusion surgery & 7 & 9 & \\
\hline \multicolumn{4}{|l|}{ Duration of surgery (min) } \\
\hline Mean \pm SD & $140.5 \pm 79.4$ & $145.5 \pm 89.4$ & 0.78 \\
\hline \multicolumn{4}{|l|}{ Blood loss (ml) } \\
\hline Mean \pm SD & $44.4 \pm 109.8$ & $51.0 \pm 129.1$ & 0.178 \\
\hline \multicolumn{4}{|c|}{ Post-operative stay et surgical unit (D) } \\
\hline Mean \pm SD & $5.1 \pm 1.2$ & $9.1 \pm 7.1$ & 0.002 \\
\hline Complications of surgery (n) & 3 & 6 & 0.087 \\
\hline
\end{tabular}


clinicians. If all previously mentioned methods (including image guided biopsies) fail then definitive diagnosis can be obtained by surgical methods.

The introduction of effective AAT in the 1950s superseded surgery as primary TB treatment (11). The need for surgery became limited to the treatment of TB complications and to diagnose uncertain lesions or otherwise unrecognized disease (12).

During the last two decades the incidence of MDR-TB increased and the number of patients treated with immunosuppressants rose as well $(9,12)$. While the threat of MDR-TB has not yet been significantly reflected in the Czech Republic, the number of people taking immunosuppression and undergoing thoracic surgery continues to increase each year (2).

The objective of diagnostic surgical procedures includes the exploration of the pleural space, evacuation of persistent or recurrent massive pleural effusion and identifying of pulmonary lesions (9).

Recurrent pleural effusion can be resolved by minimally invasive surgery - video-assisted thoracoscopic surgery. During the last two decades, the worldwide experience with VATS has shown that it is a safe and effective procedure to diagnose pleural pathology $(12,14,15)$. Microbiological analysis of the fluid alone may not confirm the diagnosis. Therefore, biopsy of the parietal pleura is a necessary part of the surgical procedure.

Another advantage of surgery is its therapeutic solution for cases where the pleural effusion is diagnosed as thoracic empyema stage II or III. In selected cases a surgical drainage, pleurectomy and decortication procedure can be performed under the same anaesthesia $(12,15)$. There have been studies reporting that tuberculous empyema was successfully managed with VATS decortication (16-18). Tuberculous empyema stage III was found in $56 \%$ of our patients with tuberculous pleural effusion. Cases with advanced empyema were converted to open surgery. Drainage and lung decortication via thoracotomy were then performed.

The surgical approach in lung nodule diagnostic procedures is a non-anatomical wedge resection with a disease-free resection line. This is an undisputed requirement for lung malignancy as well as for TB surgery. Currently the VATS is a method of choice for lesions no larger than $5 \mathrm{~cm}$, with all benefits of the minimally invasive surgery (20-22).

The coexistence of pulmonary tuberculoma with primary or metastatic lung malignancy is not rare and has been reported in $4 \%$ to $25 \%$ of all patients with TB. The coexistence of active TB and lung cancer is thought to be uncommon (23-25). However, $9.1 \%$ of our patients had synchronous TB and primary lung malignancy.

Although, there has been a continuous downward trend of TB incidence in the Czech Republic, this study identified continuously increasing number of TB incidence among surgical patients. While the cause of this occurrence remains unclear, one theoretical explanation can be linked to the use of low-dose CT screening that is capable to detect indeterminate small-volume lesions. Suspicious, non-verified, solitary or low numerous lesions of the lung parenchyma pose the double challenge of diagnosis and therapy $(8,9)$. In Western and Central Europe, USA and Japan a newly recognized indeterminate solitary lung nodule has $>80 \%$ chance of being a malignant tumor $(9,19)$. Therefore, subclinical forms of TB are detected in effort to identify the earliest stages of cancer. In the context of prevention, this increased incidence of TB in our cohort should be pointed out to give more attention in the differential diagnosis for indeterminate lung nodules, mass or pleural effusions.

Socioeconomics characteristics in our study group can be divided into 2 different subgroups: one is represented by foreign workers who show typical TB risk factors - poor living and social conditions, stressful situations, poor or weak social background, frequent use of alcohol. The group of Czech patients did not differ from the general population (2). In this group, pharmacological immunosuppression and a history of malignancies represent a risk factor.

The surgical diagnosis of TB is followed by the isolation of the patient and treatment with anti-tuberculosis agents and screening of persons at risk of TB in accordance with the valid legislation (Law on Public Health Protection and the Decree of the Ministry of Health of the Czech Republic) (4). There is currently an intense debate on the future possibility of home TB treatment instead of isolation of infected patients.

This research, however, is subject to several limitations. The possible limitation of this study may be a potential bias, which may be due to the long duration of the study - a seven-year observation interval. The lowest age of patient was 19 years, as the DTS treats adult patients only. The DTS is located in the capital city, which can affect the overall incidence.

\section{CONCLUSIONS}

TB continues to present a significant medical challenge and poses serious threat to global health. Surgical intervention has been the key in management of TB for a long time, and even nowadays remains an important diagnostic tool particularly for patients with nonobvious cases of TB when combined with high risk of malignancy.

The increasing number of immunosuppressed patients in addition to the increasing population of patients with history of oncological disease sets a new challenge for surgical diagnoses and treatments. However, in most of the diagnostic interventions the development of advanced instruments and technologies allows for the use of minimally invasive procedures.

\section{Conflict of Interests}

None declared

\section{Acknowledgements}

The study was endorsed with Institutional support MO1012.

\section{REFERENCES}

1. World Health Organization. Global tuberculosis report 2018. Geneva: WHO; 2018.

2. Institute of Health Information and Statistics of the Czech Republic. Basic overview of tuberculosis epidemiology in the Czech Republic in 2017. Prague: Czech Health Statistics; 2018.

3. Vašákova M, Hricíkova I, Kopecká E. Current approach to evaluation and treatment of tuberculosis. Remedia. 2016;26(3):236-41. (In Czech.)

4. Bártů V. Tuberculosis - infectious disease in 21st century. Med Praxi. 2008;5(6):245-8.

5. Hytych V, Horazdovsky P, Stehlik L, Pracharova S, Pohnan R, Lefnerova $\mathrm{S}$, et al. Our own method of fixation of biodegradable tracheal stent. Bratisl Lek Listy. 2015;116(5):340-2. 
6. Crofton J. Karel Styblo: a personal tribute. Bull Int Union Tuberc Lung Dis. 1991;66(4):211-3.

7. Rosenblatt MB. Pulmonary tuberculosis: evolution of modern therapy. Bull N Y Acad Med. 1973;49(3):163-96.

8. Pomerantz M, Mitchell JD. Surgery for the management of Mycobacterium tuberculosis and nontuberculous mycobacterial (environmental) infections of the lung. In: Shields TW, LoCicero J, Reed CE, Feins RH, editors. General Thoracic Surgery. 7th ed. Philadelphia: Lippincott Williams \& Wilkins; 2009. p. 1149-58.

9. Molnar TF. Tuberculosis: mother of thoracic surgery then and now, past and prospectives: a review. J Thorac Dis. 2018;10(Suppl 22):S2628-42.

10. Odell JA. The history of surgery for pulmonary tuberculosis. Thorac Surg Clin. 2012;22(3):257-69.

11. Leeming-Latham C. Unravelling the 'tangled web': chemotherapy for tuberculosis in Britain, 1940-70. The William Bynum prize essay. Med Hist. 2015;59(2):156-76.

12. Pohnan R, Ryska M, Hytych V, Matej R, Hrabal P, Pudil J. Echinococcosis mimicking liver malignancy: a case report. Int J Surg Case Rep. 2017;36:55-8.

13. Hazelrigg SR, Nunchuck SK, LoCicero J, 3rd. Video Assisted Thoracic Surgery Study Group data. Ann Thorac Surg. 1993;56(5):1039-43; discussion 1043-4.

14. Rocco G, Martucci N, La Manna C, Jones DR, De Luca G, La Rocca A, et al. Ten-year experience on 644 patients undergoing single-port (uniportal) video-assisted thoracoscopic surgery. Ann Thorac Surg. 2013;96(2):4348.

15. Yim APC, Lee TW, Izzat MB, Wan S. Place of video-thoracoscopy in thoracic surgical practice. World J Surg. 2011;25(2):157-61.

16. Barbetakis N, Paliouras D, Asteriou C, Tsilikas C. eComment: the role of video-assisted thoracoscopic surgery in the management of tuberculous empyemas. Interact Cardiovasc Thorac Surg. 2009 Mar;8(3):337-8.
17. Olgac G, Yilmaz MA, Ortakoylu MG, Kutlu CA. Decision-making for lung resection in patients with empyema and collapsed lung due to tuberculosis. J Thorac Cardiovasc Surg. 2005 Jul;130(1):131-5.

18. Subotic D, Lardinois D, Hojski A. Minimally invasive thoracic surgery for empyema. Breathe (Sheff). 2018;14(4):302-10.

19. Pfannschmidt J, Schönfeld N. Interdisciplinary treatment of patients with pulmonary tuberculosis. Zentralbl Chir. 2017;142(S 01):S53-65. (In German.)

20. Xia Z, Qiao K, He J. Recent advances in the management of pulmonary tuberculoma with focus on the use of tubeless video-assisted thoracoscopic surgery. J Thorac Dis. 2017;9(9):3307-12.

21. Luh SP, Liu HP. Video-assisted thoracic surgery-the past, present status and the future. J Zhejiang Univ Sci B. 2006;7(2):118-28.

22. Yen YT, Wu MH, Lai WW, Chang JM, Hsu IL, Chen YY, et al. The role of video-assisted thoracoscopic surgery in therapeutic lung resection for pulmonary tuberculosis. Ann Thorac Surg. 2013;95(1):257-63.

23. Bordignon V, Bultrini S, Prignano G, Sperduti I, Piperno G, Bonifati C, et al. High prevalence of latent tuberculosis infection in autoimmune disorders such as psoriasis and in chronic respiratory diseases, including lung cancer. J Biol Regul Homeost Agents. 2011;25(2):213-20.

24. Fan WC, Ting WY, Lee MC, Huang SF, Chiu CH, Lai SL, et al. Latent TB infection in newly diagnosed lung cancer patients: a multicenter prospective observational study. Lung Cancer. 2014; 85(3): 472-8.

25. Morales-García C, Parra-Ruiz J, Sánchez-Martínez JA, Delgado-Martín AE, Amzouz-Amzouz A, Hernández-Quero J, et al. Concomitant tuberculosis and lung cancer diagnosed by bronchoscopy. Int J Tuberc Lung Dis. 2015;19(9):1027-32.

Received April, 122019 Accepted in revised form October, 102019 